\title{
"Unless one does the research, it may seem as just a useless battery-consuming app" - Field Notes on COVID-19 Contact Tracing Applications
}

BORCE STOJKOVSKI, University of Luxembourg, Luxembourg

RUBA ABU-SALMA, King's College London, UK and University of California, Berkeley, USA

KAREN TRIQUET, Vrije Universiteit Brussel, Belgium

GABRIELE LENZINI, University of Luxembourg, Luxembourg

Globally, countries have been developing contact tracing applications to control the spread of the coronavirus (COVID-19) disease. In this work, we present the findings of eight focus groups we conducted with participants living in France and Germany, to explore why they decided to adopt, or not adopt, a contact tracing application as well as understand how they perceived the benefits, drawbacks, and threat model of a contact tracing application.

CCS Concepts: • Human-centered computing $\rightarrow$ User studies; $•$ Security and privacy $\rightarrow$ Social aspects of security and privacy.

Additional Key Words and Phrases: COVID-19, contact tracing, proximity tracing, human factors, user adoption

ACM Reference Format:

Borce Stojkovski, Ruba Abu-Salma, Karen Triquet, and Gabriele Lenzini. 2021. "Unless one does the research, it may seem as just a useless battery-consuming app" - Field Notes on COVID-19 Contact Tracing Applications. Digit. Threat. Res. Pract. 1, 1, Article 1 (January 2021), 17 pages. https://doi.org/10.1145/3480466

\section{INTRODUCTION}

The coronavirus (COVID-19) pandemic has led governments, health agencies, and technology companies to develop solutions to control the spread of this infectious disease. Digital contact tracing (or proximity tracing) has been proposed to help break the chain of COVID-19 infections, complement manual tracing [6], and relax lockdown restrictions. Many countries around the globe have launched national contact tracing apps that individuals can voluntarily download on their smartphones (notifying users if they have been in close proximity to someone who has tested positive for COVID19 [25]). However, the effectiveness (or utility) of these apps - whether centralized (like the French TousAntiCovid [17] based on ROBERT [5]) or decentralized (like the German Corona-Warn-App [31] based on DP-3T [34]) - highly depends on their widespread adoption by the general population.

Several user studies - mainly surveys (e.g., [2, 20, 22, 32]) - have quantitatively explored how users perceive the use and features of contact tracing apps. In this paper, we present our study, conducted in November 2020, consisting of eight focus groups with participants living in France and Germany, to qualitatively understand: (1) the factors that

Authors' addresses: Borce Stojkovski, borce.stojkovski@uni.lu, University of Luxembourg, Luxembourg; Ruba Abu-Salma, ruba.abu-salma@kcl.ac.uk, King's College London, UK, University of California, Berkeley, USA; Karen Triquet, karen.triquet@vub.be, Vrije Universiteit Brussel, Belgium; Gabriele Lenzini, gabriele.lenzini@uni.lu, University of Luxembourg, Luxembourg.

Permission to make digital or hard copies of part or all of this work for personal or classroom use is granted without fee provided that copies are not made or distributed for profit or commercial advantage and that copies bear this notice and the full citation on the first page. Copyrights for third-party components of this work must be honored. For all other uses, contact the owner/author(s).

(c) 2021 Copyright held by the owner/author(s).

Manuscript submitted to ACM

Manuscript submitted to ACM 
influence people's decision to adopt and use a contact tracing app; (2) people's perceived benefits and drawbacks of such an app; and (3) people's perceived threat/adversarial model of a contact tracing app.

Paper structure. First, we provide a short background on contact tracing apps and present related work in this domain. This is followed by an overview of the epidemiological situation in France and Germany in the second half of 2020 to better understand the context within which our study was conducted. We then describe our study design and present our findings. We conclude with a discussion and provide a set of recommendations and future work directions.

\section{BACKGROUND AND RELATED WORK}

Contact tracing is a process in which public health workers interview people who have been diagnosed with an infectious disease in an attempt to prevent onward transmission by identifying their recent contacts and isolating those who are at risk [24, 26, 27]. As the COVID-19 pandemic has raged, a wide range of contact tracing smartphone apps have been developed to control the spread of the virus [23] and supplement manual contact tracing. The purpose of these apps is to identify and notify people of potential exposure to COVID-19 in an effort to keep infection rates low while allowing greater freedom of movement. Some apps have been designed by a small number of researchers and coders, while others jointly by states, medics, and tech companies like Google and Apple [3].

By the end of March 2020 and the beginning of April 2020, many countries started to deploy contact tracing apps to combat the spread of the pandemic. However, national and local preferences have differed across countries in terms of app design, data storage, involvement of research institutes and technology companies in the app design and development stages, and adoption rate. For example, several European countries including Austria and Germany decided to deploy contact tracing apps using the Bluetooth technology and following the decentralized approach, whereas other countries like China and Singapore chose the centralized approach [14, 23]. In some other countries, public health authorities released apps using Apple and Google's Exposure Notification API [23]. The use of contact tracing apps was mandatory in some countries and voluntary in others (partially explaining the low adoption rate of some contact tracing apps).

Despite the influx of contact tracing apps, very little was known about their societal impact: how many people will download and use these apps? What will make people continue using them? How widely used do the apps have to be in order to succeed? How do people perceive the benefits and drawbacks of such apps? Are there any cross-cultural differences in people's perceptions, expectations, and privacy concerns with regard to these apps?

Online surveys. Prior work has examined public opinion of contact tracing smartphone apps. Zhang et al. conducted one of the earliest survey studies (2,612 participants) between March 30 and April 1, 2020, to explore Americans' perceptions of privacy and surveillance during the COVID-19 pandemic [38]. Preliminary results showed that Americans preferred the use of manual tracing and traditional health screenings over contact tracing smartphone apps. Abeler et al. conducted a survey of 1,055 respondents residing in the UK in March 2020 (just before the first lockdown was imposed in the UK and before any contact tracing app was deployed) [1]. About three-quarters of respondents said they would definitely or probably download and use a contact tracing app - though some respondents cited the following as barriers to adoption: risk of government surveillance and fear of user's phone being compromised. Further, Altmann et al. conducted a survey of 5,995 respondents recruited from five countries (France, Germany, Italy, the UK, and the US) between March 2020 and April 2020, to measure public support for contact tracing apps [2]. Although none of the respondents had used a contact tracing app in the past, German and US respondents were less supportive of contact tracing apps compared to respondents from other countries - citing security and privacy concerns. Using a study Manuscript submitted to ACM 
similar to that of Altmann et al., Kostka and Habich-Sobiegalla studied public acceptance of contact tracing apps in China, Germany, and the US in June 2020; they also found that respondents recruited from Germany and the US were much less accepting of using a contact tracing app than those recruited from China [21]. Other similar survey studies have been conducted [19, 20, 22, 33, 35]. Trang et al., for instance, collected data from 518 participants in Germany between April 20 and 24, 2020 investigating how the intention to install contact tracing apps is influenced by different app specifications, focusing on three dimensions: benefit appeal, privacy design, and convenience design [33]. They suggested that in order to achieve mass acceptance, policy makers should categorize a country's population into three major groups: critics, undecided, and advocates. Depending on where the majority falls, policy makers should employ corresponding strategies; for example, communicating societal-benefit appeals and ensuring minimal privacy risks are paramount for critics; for the undecided group, "convenience in app usage" is more important than highlighting privacy risks, while societal-benefit appeals should be stressed even more.

Longitudinal studies. Garrett et al. examined public opinion over time in several countries (including Australia, Germany, and the UK). They periodically recruited and surveyed respondents from these countries "in waves". Garrett et al. found that despite the widespread acceptance of contact tracing apps in Australia, download rates were lower than what was predicted [13]. Simko et al. deployed a sequence of online surveys (100 respondents recruited from various countries around the world per survey) as part of a longitudinal study [32]. They found that a significant minority of the respondents had reservations about downloading and using a contact tracing app even in the best possible privacy scenario (i.e., when a contact tracing app would provide "perfect data protection").

Most user studies conducted to explore people's privacy perceptions of and attitudes toward contact tracing smartphone apps have been quantitative studies (mainly surveys). Furthermore, they captured intentions to install or adopt a contact tracing app as a proxy for actual behavior. Our work builds on prior work by providing qualitative insights into why people decide to use, or not use, the official contact tracing app in their country of residence, how they view the benefits and drawbacks of the app, and what security and privacy concerns they have. Our study is also a cross-cultural one (Germany and France) conducted in a focus group setting, allowing participants to discuss and examine any similarities and differences in their perceptions and expectations.

\section{CONTEXTUAL INFORMATION}

\subsection{Epidemiological situation in France and Germany}

Following a decline in the number of confirmed cases and the associated morbidity and mortality rates, which in most European countries peaked in April and early May of 2020, the summer months of 2020 saw a relaxation of the stringent lockdowns and restrictions imposed during the first wave of the pandemic [9]. The second wave, however, emerged as much more disastrous with the number of deaths mounting even in Germany [29], which was largely perceived as a country that was successfully tackling the pandemic at its onset [8]. By late October 2020, a dramatic increase in the number of confirmed infections (as illustrated in Figure 1) as well as hospital and emergency unit admissions could be observed, leading to a renewed imposition of tighter control measures in various European countries [9].

In an effort to tackle the new surge of COVID-19 in France, a second nationwide lockdown began on October 30, 2020 [16]. While it was characterized by a higher number of exceptions in comparison to the first lockdown, such as schools and certain sectors remaining open, all non-essential stores and activities were again closed, working from home was requested whenever possible, and restrictions on movement were reimposed. Consequently, any activity requiring people to leave their homes, such as accessing essential services, food shopping, or outdoor exercising had to 


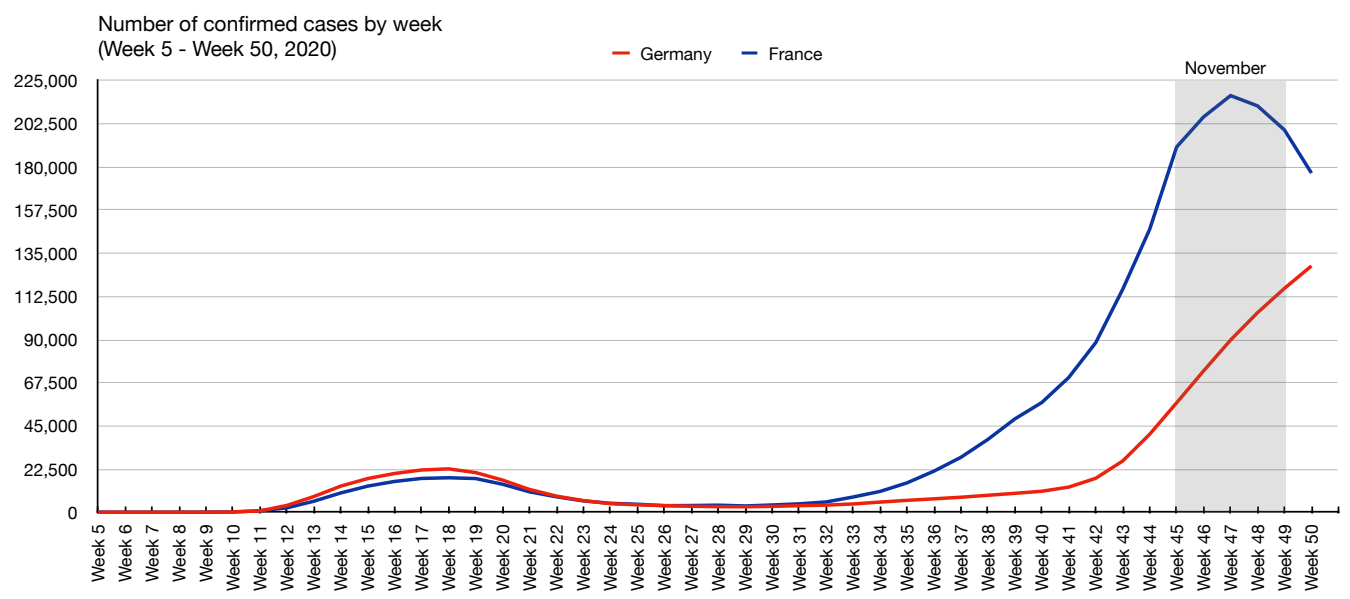

Data source: European Centre for Disease Prevention and Control 2021, www.ecdc.europa.eu

Fig. 1. Overview of the epidemiological situation in France and Germany in 2020.

be declared in a specific form called Certificate of Travel Restriction Waiver (Attestation de déplacement dérogatoire) that had to be carried at all times [18]. 38\% of the people polled by YouGov in November 2020 thought that France handled the pandemic very or somewhat well [36].

In response to the spiraling coronavirus crisis in Germany, a partial lockdown was imposed on November 2, 2020 [12]. While being a softer version of the restrictions imposed during the first wave, the partial lockdown, nevertheless, entailed closure of most non-essential premises, restrictions on meeting in public spaces, and limits on the number of people allowed to visit someone else's home. Unnecessary travel was strongly discouraged, while working from home was expected to be the norm. Regarding people's perceptions of the German government's handling of the pandemic, in mid-November 2020, 55\% of the people polled by YouGov thought that it was handled very or somewhat well [37].

\subsection{Contact tracing apps in France and Germany}

France. Built upon the centralized ROBERT (ROBust and privacy-presERving proximity Tracing) protocol [5], the official French contact tracing app was initially called StopCovid when it was launched on June 2, 2020. In an effort to address certain limitations and make progress with respect to the initial low adoption figures of StopCovid among the French population, the government relaunched the app based on the same technical principles, under the name TousAntiCovid on October 22, 2020, effectively replacing the previous version [28]. According to the open dataset published by the Ministry of Solidarity and Health of France [15], by November 20, 2020, the StopCovid/TousAntiCovid app was downloaded $9.26 \mathrm{~m}$ times in total (roughly by $14 \%$ of the population ${ }^{1}$ ). 48,959 people had confirmed their infection with COVID-19 using the app, while 12,901 people were notified by the app about exposure to COVID-19 [15].

Germany. Abandoning the initial plans of developing a contact tracing app based on a centralized software architecture akin to the one in France [7], the official German contact tracing app, called Corona-Warn-App, was launched on June 16, 2020 based on the DP-3T (Decentralized Privacy-Preserving Proximity Tracing) protocol [34]. As per the published facts and figures on November 20, 2020 by the federal government agency responsible for disease control and prevention

\footnotetext{
${ }^{1}$ As of 2020, population of France: $67.3 \mathrm{~m}[11]$

Manuscript submitted to ACM
} 
[30], the app was downloaded $22.8 \mathrm{~m}$ times in total (roughly by $27 \%$ of the population ${ }^{2}$ ). More than $3.9 \mathrm{~m}$ test results (both positive and negative) were communicated to users via the app, and $56 \%$ of the positive results were further shared by the users in order to alert others and break the chain of infections [30]. Corona-Warn-App was among the first three contact tracing apps in Europe to link to the EU interoperability gateway system, allowing for a seamless exchange of information between apps (and across borders) based on a decentralized architecture [10].

\section{METHODOLOGY}

To answer our research questions, we conducted eight focus group sessions with 24 participants in total (12 per country) during the second half of November 2020.

Participants. Study recruitment was conducted via Prolific, an online crowdsourcing platform ${ }^{3}$. Several control measures were set as pre-screening filters, such as current country of residence (France or Germany), desktop access, and willingness to participate in video interviews. To encourage greater representation and inclusion, eligible candidates were then provided with potential dates, so that they could register for a focus group session. Upon selecting a suitable time slot, participants were sent a link to the online session that they would take part in. Online sessions were hosted on the Webex video conferencing system and facilitated by the Miro collaborative platform ${ }^{4}$. This resulted in a total of eight focus groups (four French and four German), lasting for approximately one hour each. Sessions took place during the day and in the evening to meet participants' schedules.

Ethics. Our study was approved by our institution's ethics review panel, and informed consent was obtained from all participants. Participants' names and personal details were kept anonymous. Participants were also given the option to use a pseudonym when participating in the online session hosted on Webex and facilitated by Miro. All 62 participants that took part in the short pre-screening survey were compensated $£ 0.09$, corresponding to Prolific's fair rewarding practice of at least $£ 5.00$ (\$6.50) per hour. 24 of these participants were subsequently compensated at an hourly rate of $£ 10$ for their participation in the focus group sessions, as per Prolific's recommendations.

Focus Groups. The focus groups, which ranged in size from two to four participants, adopted a semi-structured approach based on the guide available in Appendix A. After briefly introducing contact tracing apps - supported by excerpts from the World Health Organization $(\mathrm{WHO})^{5}$ - and making sure that participants understood key terminology, we asked a series of questions.

Participants were asked to take part in a number of individual and group activities (as prompted by each question) using Miro. As depicted in Figure 2, Miro is a collaborative platform where individual projects, by default consisting of a blank canvas, can be created and shared with (anonymous) guests who can view or edit the canvas using a series of tools. For each focus group, a separate project consisting of several boards was created. Each board was dedicated to one question from our focus group guide.

The first author of this paper moderated all focus group sessions and guided participants through the questions and discussion. For each question, which was written on the top of the board, participants were asked to share and document their thoughts using color-coded notes that they attached to the board, to achieve breadth of knowledge and collect as many individual experiences as possible. This was followed by short plenary group discussions (before jumping to

\footnotetext{
${ }^{2}$ As of 2020, population of Germany: $83.2 \mathrm{~m}[11]$

3 https://www.prolific.co, retrieved August 16, 2021

${ }^{4}$ https://www.miro.com, retrieved August 16, 2021

${ }^{5}$ https://www.who.int/publications/i/item/WHO-2019-nCoV-Contact_Tracing-Tools_Annex-2020.1, retrieved August 16, 2021
} 


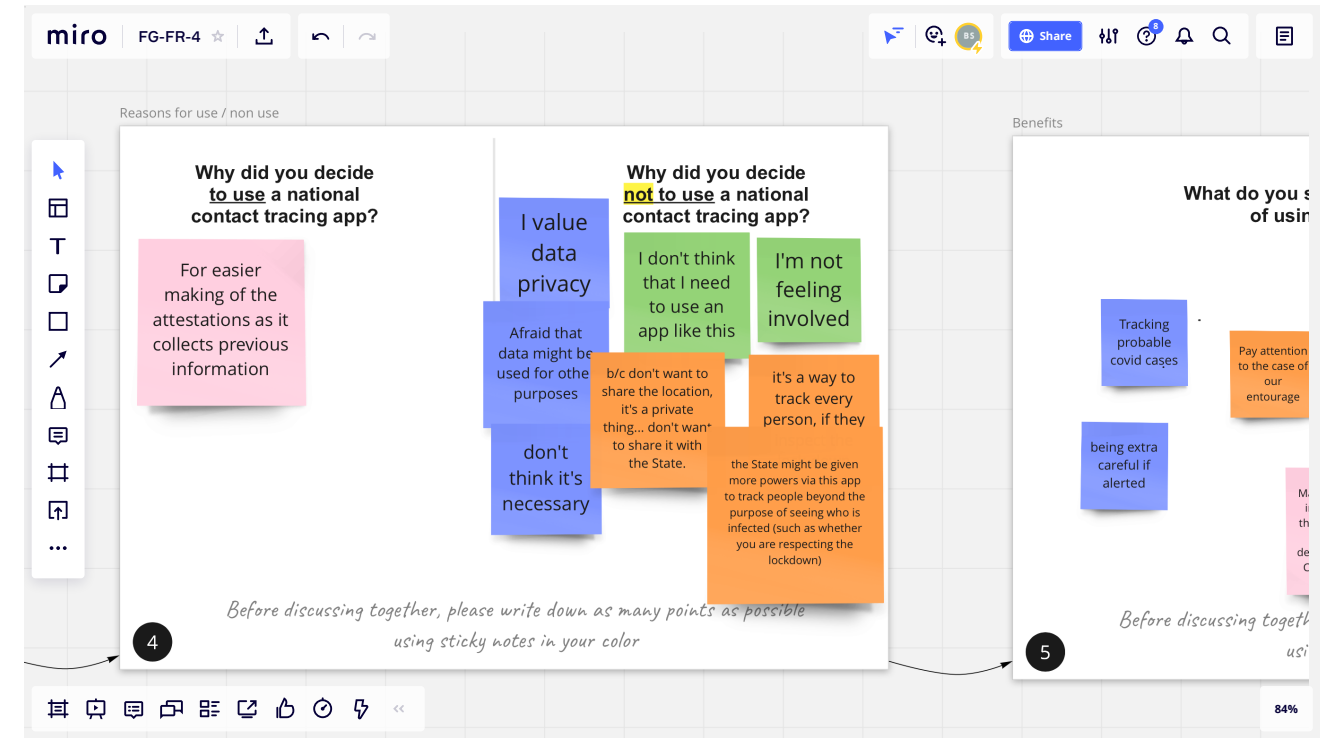

Fig. 2. The Miro online collaborative platform used in the focus group sessions

the next question), to glean insights into and explore individual experiences in depth through group discourse. Each participant was given the chance to address and build on the comments of other participants in the group. When necessary, the moderator asked individual participants direct questions to ensure all views were considered.

Data analysis. The data collected and analyzed for the purpose of this study included individually written participant answers (color-coded notes on the Miro platform) and group discussions (transcripts of Webex recordings). Session recordings were transcribed, and individual notes were exported to MAXQDA, to allow for subsequent thematic analysis. Tags denoting the French and German groups were included for all notes. The thematically-driven questions on the Miro boards were used as a guide for the creation of first-level codes (e.g., adoption/non adoption factors, benefits/drawbacks, etc.), which we used to analyze the focus group data (i.e., individual answers and discussion points). Inductive coding was then used to produce second-level codes, which we used to analyze the data in depth and formulate insights.

Preliminary coding of the data collected from one focus group session was undertaken by the lead researcher. The coding was then independently verified by a second researcher, to refine the codebook, resolve disagreements, and reach consensus about the identified coding categories. The data collected from the other three focus group sessions was then coded by the lead researcher, in consultation with the second researcher when necessary. Our qualitative codebook can be found in Appendix B.

\section{FINDINGS}

Table 1 provides an overview of our participant demographics. 
Table 1. Participant demographics. $\mathrm{N}=12$ per country.

\begin{tabular}{lrr}
\hline Demographics & France & Germany \\
\hline \hline Female & $4(33 \%)$ & $7(58 \%)$ \\
Male & $8(67 \%)$ & $5(42 \%)$ \\
\hline Median age & 30 & 31 \\
Age range & {$[20-62]$} & {$[26-54]$} \\
\hline National residents & $5(42 \%)$ & $10(83 \%)$ \\
Foreign residents & $7(58 \%)$ & $2(17 \%)$ \\
\hline Employed (full-time or part-time) & $8(67 \%)$ & $10(83 \%)$ \\
\hline Student status & $4(33 \%)$ & $2(17 \%)$ \\
\hline Currently use a national contact tracing app & $2(17 \%)$ & $8(67 \%)$ \\
Used a national contact tracing app in the past, but stopped using it & $3(25 \%)$ & $1(8 \%)$ \\
Have not used a national contact tracing app & $7(58 \%)$ & $3(25 \%)$ \\
\hline \hline
\end{tabular}

\subsection{Reasons for adoption}

The following themes were identified as dominant reasons for installing and using contact tracing apps by focus group participants. Participants included those that at the time of the study used or had previously used a contact tracing app.

5.1.1 Desire to act responsibly and exercise civic responsibility. Participants from both countries (France and Germany) viewed the adoption of contact tracing apps as a means to contribute to a greater societal cause and to help prevent the spread of coronavirus. This is depicted more clearly by the following statements:

- "to limit the propagation of the virus as efficiently as possible - a civic duty, I guess" (FR, p7)

- "helping scientists do their job" (DE, p22)

- "supporting the society" (DE, p23)

One participant viewed the adoption and use of a contact tracing app as a self-imposed duty to (positively) influence others' beliefs and behaviors in this regard: "To be a role model ... to encourage friends, colleagues to use that by themselves. Show to my kids that I want to do everything [that] I can do" (DE, p23).

5.1.2 Personal safety. Another recurring theme and key motivator for adoption was the desire to protect oneself and others, especially family members, friends, elderlies, and vulnerable people (at-risk groups):

- "because I want to know if I have been in contact with an infected person" (DE, p19)

- "to know if I am under risk" (DE, p17)

- "take precautions like self isolation if appropriate" (DE, p15)

- "to stay safe and take care of family and friends" (DE, p20)

- "protecting the parent generation" (DE, p23)

5.1.3 Security, privacy, and trust. Feeling in (partial) control of the data collected, taking an informed approach regarding the type and use of data handled, and having trust in the government and/or app developers handling the data were voiced as motivating factors that increase adoption of contact tracing apps and/or minimize users' privacy concerns.

- "A lot of warnings and options to respect the privacy of every user - seemed like a safe choice to use it" (FR, p7)

- "because they're safe when it comes to my data” (DE, p19) 
5.1.4 Other reasons. Being curious about trying out a 'new technology', evolving social dynamics (e.g., increased social interactions and the desire to avoid lockdowns), and requests to install a contact tracing app by an employer or when traveling abroad were all mentioned as reasons why participants installed and/or considered using contact tracing apps. Moreover, several participants indicated that although they preferred to voluntarily install an app, they would still install an app even if it were mandatory to do so. In contrast, others demonstrated a preference for a more mandated approach. One participant specifically voiced refusing to install a contact tracing app, precisely because it was voluntary to do so, questioning the app's reliability and placing greater trust in a more mandated approach. We describe reasons for non-adoption next.

\subsection{Reasons for non-adoption}

5.2.1 Lack of utility. A significant number of participants believed that there was no added benefit or value of existing contact tracing apps, as illustrated by these verbatims:

- "don't think it's necessary" (FR, p10)

- "I don't think that I need to use an app like this" (FR, p12)

Moreover, the second (partial) lockdown which overlapped with our focus group sessions, seemed to have impelled participants to further question the usefulness of the apps in light of remote working and more general self-isolation measures, as the following expressions highlight:

- "I do not need them because of home office" (DE, p13)

- "I have never been in the situation to use one, I'm mostly at home" (DE, p18)

This lack of perceived utility or perceived benefit was both driven by and in turn reinforced low adoption rates. Participants considered the number of current users of a contact tracing app (and how in turn they perceived its value) when making their own decisions to adopt or not adopt the app.

- "Even [if] smartphone was activated, wouldn't have done it because no one seemed to be doing it. The consensus was that it was not very efficient." (FR, p4)

- "It's still like a trial at the moment, I feel like it needs to be out... so basically, I don't" (DE, p18)

5.2.2 Lack of trust. A number of factors and entities were brought up in the context of trust. Participants mentioned that they did not necessarily trust their government, the developers of the contact tracing app, nor the technology itself.

- "the results of those apps with the underlying technology do not work as intended, especially the distance cannot be determined precisely" (DE, p13)

- "Not accurate enough due to the Bluetooth used as a technology" (FR, p5)

- "I do not trust the companies which developed the app" (DE, p13)

- "Not enough information about the editor [developer]" (FR, p3)

- "the State might be given more powers via this app to track people beyond the purpose of seeing who is infected (such as whether you are respecting the lockdown)" (FR, p9)

5.2.3 Lack of clarity and communication. Poor public communication, in terms of clarity and outreach, and lack of public engagement were issues that emerged from our focus groups and were especially discussed by French participants.

- “...[it] wasn't really explained, they said you can download it, it would be a good idea, but had no idea of what the use of this would be" (FR, p4)

Manuscript submitted to ACM 
"Unless one does the research, it may seem as just a useless battery-consuming app"

- "You don't know where and who owns the data in the end. When the government told about that application, they told it was a private company and we use Bluetooth, but we do not keep the tracing data. So it's a little bit difficult to tell." (FR, p1)

Participants also stressed the additional effort being placed on them to understand the purpose of a contact tracing app.

- "The benefits of using it are not clearly explained by the State. Unless one does the research, it may seem as just a useless battery-consuming app" (FR, p7)

The government's approach to persuading the population to start using a contact tracing app was perceived as paternalistic by some participants, which resulted in unwillingness to participate in the collective action.

- "they treat us as children so I acted as a child" (FR, p1)

In this context, participants also expressed lack of role models promoting app adoption. Some participants believed that public figures and government officials did not install contact tracing apps.

5.2.4 Poor usability. In addition to lack of utility, some participants mentioned that contact tracing apps drained their phone's battery, while others found using and interacting with the apps complicated, resulting in them not using the apps (any longer).

- "Not user friendly, they need to create something easy to understand to make it popular" (FR, p6)

5.2.5 The ostrich effect. Psychological and practical discomforts resulting from the apps' potential exposure notifications were mentioned by participants as reasons for not adopting contact tracing apps.

- "I'm afraid of what will happen if it counts me as an infected person - I prefer not knowing!" (FR, p7 quoting a friend)

Other participants explained that they did not find the current privacy-preserving mechanisms in contact tracing apps flexible or reliable enough to meet the needs of citizens who were willing to share more private data in return for more contextual information.

- "If I would use the app, I think I would have, to be frank, a psychological problem. If I get an alarm I would like to know where I put myself at risk, as I am extremely careful. I'm not carrying a normal mask, I'm wearing a gas mask, with highly-effective filters, I wear highly-effective protection glasses, I always carry gloves and I only go shopping when it is absolutely necessary. If I would get an alarm, I would be really concerned what I have done wrong and where I put myself at risk" (DE, p21)

\subsection{Benefits and Drawbacks}

The benefits highlighted by participants in the focus groups corresponded to and echoed the reasons why participants decided to adopt a contact tracing app. Reasons included helping identify and break chains and clusters of infection, identifying potential risks, and informatively deciding and planning next steps.

- "Ability to trace the possible infections scenarios objectively - when getting tested as "positive" a person often forgets to warn every single person he/she contacted" (FR, p7)

- "knowing the risk level for myself, feel rather comfortable because others will be notified if I am infected and I can't reach the people I contacted by myself" (DE, p17)

- "Knowing whether or not you should stay home/get things delivered or go out instead" (FR, p11) 
Recent improvements and additions of features in the French app, demonstrating contextual sensitivities to user needs (i.e., administrative mobility considerations during lockdown), albeit not directly linked to the act of digital contact tracing, were appreciated by participants who used the app. These voiced benefits could potentially mobilize people to adopt contact tracing apps.

- "While the former functions were just "nice to have" (just global security, but no everyday "usefulness"), the attestations management was a really nice feature for everyday use" (FR, p7)

- "[decided to use TousAntiCovid] for easier making of the attestations as it collects previous information" (FR, p11)

While making a link between perceived benefits and reasons for adoption is straightforward, and arguably the voiced themes in this section could be analyzed in conjunction with Section 5.1, here we are interested in developing a better understanding of the benefits highlighted by participants who had not used a contact tracing app before.

Unsurprisingly, even participants who had not used contact tracing apps agreed to a great extent with the potential benefits of these apps.

- "To know if you have got the virus or if you're exposed to" (FR, p3)

- "help scientists get enough data about the pandemic and the spreading behavior of the virus" (DE, p24)

- "I discovered that we can complete attestations faster, [I could] globally gain some time" (FR, p12)

Nevertheless, there were some participants, albeit a very small number, that did not see any benefits of these apps personally or whatsoever.

- "for really frightend people this could help to ease their minds or to give them the feeling of security" (DE, p13)

- "I will not have the app to tell me, my friend or family if they have covid will just tell me" (FR, p9)

- "searching for the benefits... but I couldn't figure it" (FR, p6)

As for drawbacks, participants who used the French and German apps highlighted a number of shortcomings with regard to what and how apps communicated information to users as well as the general app design. Most participants touched upon the fact that current apps provided insufficient procedural information and did not offer personalized and useful information (e.g., personal risk levels).

- "People don't know what to do with certain warning levels" (DE, p14)

- "somehow quantify my personal risk better, but result is poor, no personal gain, only confusion (by usage, by information about risks)" (DE, p23)

Further, contact tracing apps were perceived as lacking interactivity. In this regard, participants offered their input and suggested the design of features that would address some of their unmet needs.

- "Feature request: to receive the latest updates about the pandemic and specific to my area" (DE, p18)

- "It would be nice to have a list of places where you can go and get a test, news about new developments on the pandemic situation, list of special places that are crowded and not to be visited etc." (DE, p17)

Our participants also mentioned costs and expenses, such as the usage of own mobile data plans, device data storage, and battery drainage as drawbacks they were concerned about.

Another issue mentioned by participants revolved around the poor implementation of a Test-Trace-Isolate strategy, which in some cases could render contact tracing apps useless.

- "problem is also the accuracy, who did really have it... maybe i'm positive but i never tested, because there is not enough hospital staff to do it nor test itself is accurate enough" (FR, p6)

- "the data is a few days old, so if you're infected, you could already have been a risk for others" (DE, p19) Manuscript submitted to ACM 
"Unless one does the research, it may seem as just a useless battery-consuming app"

Finally, some of the drawbacks mentioned referred to the security and privacy aspects of contact tracing apps.

- "in theory it could be tracked where I have been, probably through the contacts but I think that is unlikely" (DE, p15)

- "I can see why there would be privacy concerns (because it asks for your location), but since I was in other countries that utilize COVID19 applications, it didn't bother me anymore (due to priming)." (FR, p11)

\subsection{Privacy concerns and threats}

One of the most prominent privacy concerns particularly raised by participants residing in France was location tracking. This was mentioned among other concerns with regard to the power that governments and involved parties could exert by gathering large amounts of data through the app.

- "I think they ask for too much information" (FR, p2)

- "the government can track the people and abuse the data" (FR, p5)

- "it invades my privacy and I don't want the state to know where I am and who I am with" (FR, p7 quoting a friend)

- "the government institutions could learn my activities, my habits, maybe even commercial vendors, difficult to know..." (FR, p5)

These are valid concerns taking into consideration the many unknowns and ongoing efforts to find and fix vulnerabilities in this domain of security and privacy research [4]. Nevertheless, participant discussions shed light on potential misunderstandings of the technicalities of contact tracing (i.e., exhibiting examples of folk models). We also speculate that participant discussions about geophysical location tracking might as well be due to specific (mis)understandings of how digital contact tracing apps work and what the apps' actual adversarial model is. Yet, some participants did not express any privacy concerns in this specific context.

- "Not really concerned about "privacy" of my geolocation and interaction data, especially for a state-backed app" (FR, p7)

- "not really... the government can find a lot of things about me or anybody already, they don't need this sort of app for that info, so not concerned" (FR, p4)

As for adversaries, our participants mostly mentioned their government followed by app developers, referring to companies behind app development and not individual programmers. In this regard, participants were mostly concerned about either not having sufficient levels of trust to start with, hindering adoption by default, or experiencing negative outcomes as a result of erosion of trust which was placed in the collective response to the pandemic.

- "I don't want anyone to know about my location history. I am willing to share this in the app only to protect myself and others but without the individual details. If the government, or app developers, any company, any third party could possibly know about this for any reason other than covid-tracing I would be very uncomfortable." (DE, p17)

\section{DISCUSSION}

\subsection{Summary of results}

Our findings suggest that, quite often, participants demonstrated a sense of civic responsibility and willingness to adopt contact tracing apps. However, our findings also show that miscommunicating the purpose and benefits of contact tracing apps, insufficient user inclusion (in the app design process), and lack of transparency (with regard to app functionality and data collection and processing) are factors that hinder the adoption of contact tracing apps. 
The investigation into perceived benefits and drawbacks of contact tracing apps echoed and served to further reinforce why participants might have chosen to adopt/not adopt their country's official contact tracing app. The intent to contribute to a more societal mission (i.e., identifying risks and exposure) was most prominent in the discourse. Instances of participants finding personal value and/or daily relevance of the app (e.g., easing the attestation process) were seen as beneficial. In contrast, lack of personalization in current apps, poor interactivity, and lack of communication were expressed as drawbacks impacting perceived relevance and usefulness.

Ultimately, participants did voice concerns regarding the possible risks of location tracking as well as the placement of trust in enabling the collection and creation of rich individual data profiles that could potentially be misused. Nevertheless, these privacy concerns and perceived threats seemed to be mostly overshadowed by other needs across the focus groups. This raises the question as to whether the perceived benefits and usefulness of contact tracing apps are on par with, if not even more important than, privacy-preserving features when it comes to adoption.

\subsection{Similarities and differences between and within study groups}

In an effort to better understand and represent the different perspectives that were expressed during the sessions as well as to position similar views in relative proximity to each other, we divided our study participants into four groups (A, B, C, and D), as depicted in Figure 3.

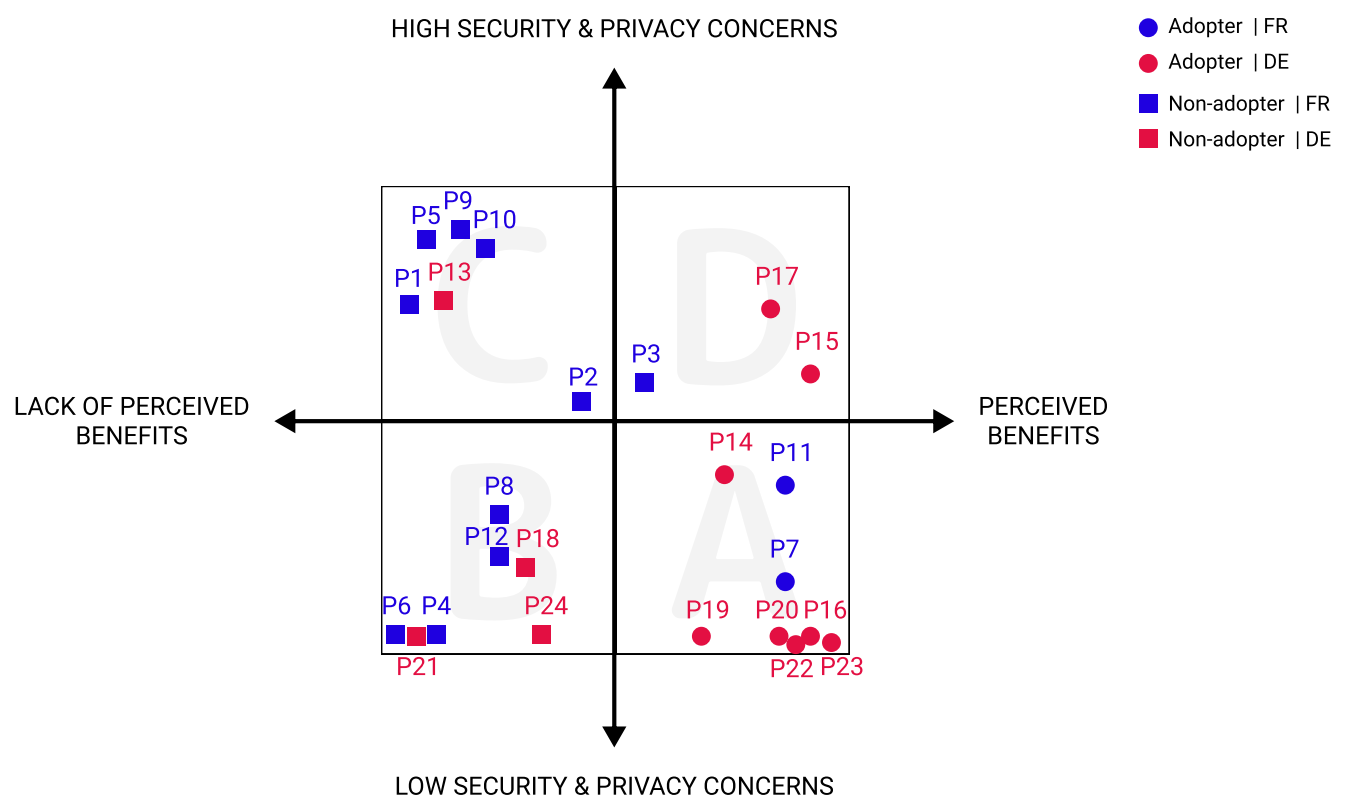

Fig. 3. Affinity mapping of focus group participants based on their responses

French participants that used a contact tracing app are represented using a blue circle next to their ID. German adopters are represented using a red circle. Those that had not adopted an app are represented using a square next to their ID, in blue and red, to denote French and German non-adopters, respectively. The two axes divide the space along the dimensions of $(x)$ the perception of benefits and $(y)$ security \& privacy concerns.

Manuscript submitted to ACM 
Group A The bottom-right quadrant consists solely of adopters of contact tracing apps that have low security and privacy concerns, and for whom the perceived benefits of contact tracing apps outweigh any drawbacks by far.

Group B The bottom-left region gathers non-adopters who, despite having low or moderate security and privacy concerns, decided not to adopt a contact tracing app because they do not perceive the benefits or added values of the available contact tracing app in their country or in general.

Group C The upper-left quadrant gathers those participants that have moderate to high concerns about security and privacy associated with the usage of contact tracing apps. Furthermore, they either do not see the benefits of using such apps, or for them the drawbacks outweigh any benefits.

Group D The upper-right quadrant consists of both adopters and non-adopters. Both groups share moderate security and privacy concerns; however, for the first group, the perceived benefits associated with using a contact tracing app outweigh any drawbacks or concerns. For the latter, the perceived benefits are not there yet for them to decide to adopt or continue using a contact tracing app.

Looking at the distribution of our small sample of French and German participants across the four groups, we can observe that German study participants mostly fall in Group A; i.e., they clearly saw the benefits of using contact tracing apps and they expressed low to moderate security and privacy concerns. In contrast, the lack of perceived benefits was overwhelming among the French study participants, who are divided into two equal sets based on the level of concern with respect to security and privacy.

Contextualizing the adoption trend observed in our focus groups using the official adoption figures of the German and French contact tracing apps reported in November 2020 (see Section 3.2), we can see that our findings are in line with the higher adoption figures in Germany compared to France at the time of the study. Interestingly, this contrasts with the cross-cultural investigations of Altmann et al. [2] who reported that intentions to use contact tracing apps among Germans would be lower due to security and privacy concerns.

While this is certainly a question worthy of further exploration, we speculate that the following factors could have contributed to the higher adoption rate among Germans:

- A public debate on the benefits and drawbacks of centralized and decentralized approaches to contact tracing, contributing to the decentralized architecture of the Corona-Warn-App and the discourse surrounding it. For instance, German participants reported that it was common knowledge that in Germany a lot of emphasis is placed on data protection. Thus, while the majority of our German participants did not express security and privacy concerns, this should not be interpreted as if they had no concerns in general, but rather that they did not have these concerns with regard to Corona-Warn-App.

- The higher levels of trust in the government and positive perceptions of the government's handling of the crisis. According to Kostka and Habich-Sobiegalla, whether or not people have trust in the government has a powerful impact on the acceptance of contact tracing apps in Germany [21].

- The perception of a streamlined and clear public communication of the app's role and benefits as part of the collective response to the pandemic, including deep integration in the country's TTI strategy.

Looking at Group B, we can observe that for a number of French and German participants, security and privacy concerns are not the (primary) obstacle to adoption. To make these participants become adopters, both countries would need to work on the value proposition relevant to this potential user group. While some citizens may benefit from clear, targeted, and streamlined communication of the benefits and ways to get onboarded, others may be motivated to join only after a significant proportion of the population is already using a contact tracing app, which could potentially 
ease some of their doubts about effectiveness and accuracy. In this regard, additional useful features, such as the travel restriction waiver certificate (attestation) in France or local and personalized information could potentially further increase adoption.

While citizens that fall in Group C appear to be most resistant to adoption of contact tracing apps, some might decide to adopt, provided that the benefits outweigh their perceived drawbacks, and their concerns with regard to security and privacy are addressed or alleviated. To this end, the app's usefulness and effectiveness need to be evident, and trust needs to be established and nurtured. If we assume that this group corresponds to what Trang et al. have categorized as critics [33], emphasis should particularly be placed on the societal benefits of contact tracing apps. Nevertheless, as highlighted by Utz et al., we should expect that a certain number of people would be unwilling to adopt the app [35].

The question of trust is particularly important also for adopters in Group D, who are likely to discontinue using a contact tracing app should the trust they placed be eroded. To ensure that this does not happen, all stakeholders involved in the development, maintenance, and operation of the contact tracing app need to apply appropriate cybersecurity measures and practice the highest ethical standards at all times.

Limitations. Our study has limitations that can be broadly attributed to the collection of self-report data, which does not necessarily reflect actual behavior and the fast-tracked nature of the research conducted to inform the current and future design of contact tracing apps. That said, we aimed - by conducting our focus groups - to elicit diverse views and opinions, by offering flexible interview schedules and minimizing the use of Prolific's prescreening filters to recruit participants. However, our focus groups were conducted in English, which might have impacted how participants expressed themselves (if English was not their first language). Further, as opposed to Prolific, in-situ and multi-channel targeted recruitment approaches could have allowed us to reach out to a diverse sample of participants, based on age, profession, and digital literacy, ensuring that different user profiles and risk groups were explicitly accounted for.

Due to the COVID-19 pandemic, we are aware that our study design and online participant recruitment approach inevitably excluded a large portion of participants and made our findings less generalizable. Further, participating in sessions in a hybrid fashion via Webex and using Miro to share thoughts, in contrast with a traditional in-situ focus group discussion, might have required extra effort from participants who lacked prior experience with those communication and collaboration platforms. While many welcomed this novel participatory approach, on a few occasions we had to assist participants in attaching their notes or navigating Miro. In some cases, this might have got in the way of gleaning additional insights into our investigation.

While we cannot exclude the possibility of social desirability bias toward the moderator and other focus group participants, we believe that the wide range of responses and preferences expressed by our participants indicate that heterogeneity of views was acquired. Furthermore, we did not observe tendencies of participants to take over the discussion or devalue the opinions of others, and we ensured to offer every participant a chance to express their views.

Future Work. Future large-scale quantitative studies would enable greater generalizability of our findings. Future work should also consider investigating the potential differences with respect to people's opinions of centralized and decentralized approaches. In this regard, our focus groups aimed to account for a balanced view (centralized and decentralized) to answer our research questions. Furthermore, the unique socio-technical differences that different countries may have to deal with to understand societal uptake are worth noting. Several differences were visible in our focus groups among French and German participants, but those would require more systematic analysis for app deployment and uptake.

Manuscript submitted to ACM 


\section{CONCLUSION}

Considering human factors when exploring the adoption as well as the security and privacy aspects of COVID-19 contact tracing apps is a timely societal challenge. Our work aims to provide greater granularity and understanding of the current more abstract quantitative investigations of contact tracing app adoption and use. Our findings complement ongoing efforts from a socio-technical lens, given the complex and interdisciplinary needs required to overcome this shared reality.

Our field notes also serve to highlight future recommendations for practice and policy. There is a broad spectrum of user needs that contact tracing apps should take into consideration, in order to enhance uptake. Therefore, we believe that it would be useful to explore and define "archetypes" reflecting the diverse decisions that people make to adopt and use contact tracing apps. These in turn could be responsively leveraged to provide targeted, clear, and dedicated communication pathways within and beyond the app (policy and practice action), as well as integrated functionalities and design features (practice action).

\section{ACKNOWLEDGMENTS}

We thank Nataliia Bielova and Iraklis Symeonidis for the fruitful discussions we had during the early stages of this project. We also thank our anonymous reviewers for their useful comments and suggestions on how to improve this work. Authors are supported by the Luxembourg National Research Fund: PRIDE15/10621687/SPsquared and COVID-19/2020/1/14732977-Legafight grants.

\section{REFERENCES}

[1] Johannes Abeler, Sam Altmann, Luke Milsom, Séverine Toussaert, and Hannah Zillessen. 2020. Support in the UK for app-based contact tracing of COVID-19. (March 26, 2020). Retrieved August 7, 2021 from https://osf.io/huqtr/.

[2] Samuel Altmann, Luke Milsom, Hannah Zillessen, Raffaele Blasone, Frederic Gerdon, Ruben Bach, Frauke Kreuter, Daniele Nosenzo, Séverine Toussaert, and Johannes Abeler. 2020. Acceptability of App-Based Contact Tracing for COVID-19: Cross-Country Survey Study. FMIR Mhealth Uhealth 8, 8 (28 Aug 2020), e19857. https://doi.org/10.2196/19857

[3] Apple Inc. and Google Inc. 2020. Privacy-Preserving Contact Tracing. (April 10, 2020). Retrieved August 16, 2021 from https://covid19.apple.com/ contacttracing.

[4] Lars Baumgärtner, Alexandra Dmitrienko, Bernd Freisleben, Alexander Gruler, Jonas Höchst, Joshua Kühlberg, Mira Mezini, Richard Mitev, Markus Miettinen, Anel Muhamedagic, Thien Duc Nguyen, Alvar Penning, Dermot Frederik Pustelnik, Filipp Roos, Ahmad-Reza Sadeghi, Michael Schwarz, and Christian Uhl. 2020. Mind the GAP: Security \& Privacy Risks of Contact Tracing Apps. arXiv:2006.05914 [cs.CR]

[5] Claude Castelluccia, Nataliia Bielova, Antoine Boutet, Mathieu Cunche, Cédric Lauradoux, Daniel Le Métayer, and Vincent Roca. 2020. ROBERT: ROBust and privacy-presERving proximity Tracing. (May 2020). https://hal.inria.fr/hal-02611265

[6] Justin Chan, Landon P. Cox, Dean P. Foster, Shyam Gollakota, Eric Horvitz, Joseph Jaeger, Sham M. Kakade, Tadayoshi Kohno, John Langford, Jonathan Larson, Puneet Sharma, Sudheesh Singanamalla, Jacob E. Sunshine, and Stefano Tessaro. 2020. PACT: Privacy-Sensitive Protocols And Mechanisms for Mobile Contact Tracing. IEEE Data Eng. Bull. 43, 2 (2020), 15-35. http://sites.computer.org/debull/A20june/p15.pdf

[7] Deutsche Welle. 2020. In U-turn, Germany backs Google and Apple on virus app. (April 26, 2020). Retrieved August 16, 2021 from https: $/ / \mathrm{p} . \mathrm{dw} \cdot \mathrm{com} / \mathrm{p} / 3 \mathrm{bRKp}$.

[8] Heribert Dieter. 2020. Germany in the COVID-19 crisis: Poster child or just lucky? The fournal of Australian Political Economy 85 (2020), $101-107$. https://search.informit.org/doi/10.3316/ielapa.213086677935227

[9] European Centre for Disease Prevention and Control. 2020. Updated projections of COVID-19 in the EU/EEA and the UK. (November 23, 2020). Technical Report. ECDC, Stockholm.

[10] European Commission. 2020. Coronavirus: EU interoperability gateway goes live, first contact tracing and warning apps linked to the system. (October 19, 2020). [Press release], Retrieved August 16, 2021 from https://ec.europa.eu/commission/presscorner/detail/en/ip_20_1904.

[11] Eurostat. 2021. Population on 1 January [TPS00001]. Retrieved August 7, 2021 from https://ec.europa.eu/eurostat/databrowser/bookmark/6ef61f16dadc-42b1-a6ce-3ddfda4727e8?lang=en.

[12] Federal Government of Germany. 2020. Videokonferenz der Bundeskanzlerin mit den Regierungschefinnen und Regierungschefs der Länder. (October 28, 2020). [Press release 381], Retrieved August 16, 2021 from https://www.bundesregierung.de/breg-de/suche/videokonferenz-derbundeskanzlerin-mit-den-regierungschefinnen-und-regierungschefs-der-laender-am-28-oktober-2020-1805248.

Manuscript submitted to ACM 
[13] Paul M Garrett, Joshua P White, Stephan Lewandowsky, Yoshihisa Kashima, Andrew Perfors, Daniel R Little, Nic Geard, Lewis Mitchell, Martin Tomko, and Simon Dennis. 2021. The acceptability and uptake of smartphone tracking for COVID-19 in Australia. Plos one 16, 1 (2021), e0244827.

[14] Global Times. 2020. New health code raises privacy concerns. (May 26, 2020). Retrieved August 16, 2021 from https://www.globaltimes.cn/content/ 1189494.shtml.

[15] Government of France. 2020. Métriques d'utilisation de l'application TousAntiCovid. [Data set], Retrieved August 16, 2021 from https://www.data. gouv.fr/en/datasets/metriques-dutilisation-de-lapplication-tousanticovid/.

[16] Government of France. 2020. Présentation du Premier ministre sur les mesures contre la Covid-19. (October 29, 2020). Retrieved August 16, 2021 from https://www.gouvernement.fr/partage/11839-presentation-du-premier-ministre-sur-l-application-des-mesures-pour-lutter-contre-la-covid-19.

[17] Government of France. 2020. TousAntiCovid. Retrieved August 16, 2021 from https://bonjour.tousanticovid.gouv.fr.

[18] Government of France. 2021. Attestations de déplacement. Retrieved August 16, 2021 from https://www.interieur.gouv.fr/Actualites/L-actu-duMinistere/Attestations-de-deplacement.

[19] Eszter Hargittai, Elissa M. Redmiles, Jessica Vitak, and Michael Zimmer. 2020. Americans' willingness to adopt a COVID-19 tracking app. First Monday 25, 11 (Oct. 2020). https://doi.org/10.5210/fm.v25i11.11095

[20] Gabriel Kaptchuk, Daniel G. Goldstein, Eszter Hargittai, Jake M. Hofman, and Elissa M. Redmiles. 2020. How good is good enough for COVID19 apps? The influence of benefits, accuracy, and privacy on willingness to adopt. CoRR abs/2005.04343 (2020). arXiv:2005.04343 https://arxiv.org/abs/ 2005.04343

[21] Genia Kostka and Sabrina Habich-Sobiegalla. 2020. In Times of Crisis: Public Perceptions Towards COVID-19 Contact Tracing Apps in China, Germany and the US. SSRN (September 16, 2020) (2020). https://doi.org/10.2139/ssrn.3693783

[22] Tianshi Li, Jackie Yang, Cori Faklaris, Jennifer King, Yuvraj Agarwal, Laura Dabbish, and Jason I. Hong. 2020. Decentralized is not risk-free: Understanding public perceptions of privacy-utility trade-offs in COVID-19 contact-tracing apps. CoRR abs/2005.11957 (2020). arXiv:2005.11957 https://arxiv.org/abs/2005.11957

[23] MIT Technology Review. 2020. A flood of coronavirus apps are tracking us. Now it's time to keep track of them. (May 7, 2020). Retrieved August 16, 2021 from https://www.technologyreview.com/2020/05/07/1000961/launching-mittr-covid-tracing-tracker.

[24] MIT Technology Review. 2020. Five things we need to do to make contact tracing really work. (April 28, 2020). Retrieved August 16, 2021 from https://www.technologyreview.com/2020/04/28/1000714/five-things-to-make-contact-tracing-work-covid-pandemic-apple-google/.

[25] MIT Technology Review. 2020. Some prominent exposure apps are slowly rolling back freedoms. (November 23, 2020). Retrieved August 16, 2021 from https://www.technologyreview.com/2020/11/23/1012491/contact-tracing-mandatory-singapore-covid-pandemic.

[26] National Geographic. 2020. The magnitude of America's contact tracing crisis is hard to overstate. (September 1, 2020). Retrieved August 16, 2021 from https://www.nationalgeographic.com/science/article/contact-tracing-crisis-magnitude-hot-mess-america-fixes-coronavirus-cvd.

[27] NPR. 2020. COVID-19 Contact Tracing Workforce Barely 'Inching Up' As Cases Surge. (October 14, 2020). Retrieved August 16, 2021 from https://www.npr.org/sections/health-shots/2020/10/14/923468159/covid-19-contact-tracing-workforce-barely-inching-up-as-cases-surge.

[28] Radio France Internationale. 2020. France rolls out new Covid tracking app 'TousAntiCovid'. (October 22, 2020). Retrieved August 16, 2021 from https://www.rfi.fr/en/france/20201022-france-rolls- out-new-covid-19-mobile-tracking-app-tous-anti-covid-stopcovid.

[29] Robert Koch Institut. 2020. Coronavirus Disease 2019 (COVID-19) Daily Situation Report of the Robert Koch Institute. (November 15, 2020). Retrieved August 16, 2021 from https:/www.rki.de/DE/Content/InfAZ/N/Neuartiges_Coronavirus/Situationsberichte/Nov_2020/2020-11-15-en.pdf.

[30] Robert Koch Institut. 2020. Kennzahlen zur Corona-Warn-App. (November 20, 2020). Retrieved August 16, 2021 from https://www.coronawarn.app/ assets/documents/2020-11-20-cwa-daten-fakten.pdf.

[31] Robert Koch Institut. 2020. Open-Source Project Corona-Warn-App. Retrieved August 16, 2021 from https://www.coronawarn.app.

[32] Lucy Simko, Ryan Calo, Franziska Roesner, and Tadayoshi Kohno. 2020. COVID-19 Contact Tracing and Privacy: Studying Opinion and Preferences. CoRR abs/2005.06056 (2020). arXiv:2005.06056 https://arxiv.org/abs/2005.06056

[33] Simon Trang, Manuel Trenz, Welf H Weiger, Monideepa Tarafdar, and Christy MK Cheung. 2020. One app to trace them all? Examining app specifications for mass acceptance of contact-tracing apps. European fournal of Information Systems 29, 4 (2020), 415-428. https://doi.org/10.1080/ 0960085X.2020.1784046

[34] Carmela Troncoso, Mathias Payer, Jean-Pierre Hubaux, Marcel Salathé, James Larus, Edouard Bugnion, Wouter Lueks, Theresa Stadler, Apostolos Pyrgelis, and Daniele Antonioli. 2020. DP-3T: Decentralized Privacy-Preserving Proximity Tracing. Retrieved August 16, 2021 from https: //github.com/DP-3T/documents.

[35] Christine Utz, Steffen Becker, Theodor Schnitzler, Florian M. Farke, Franziska Herbert, Leonie Schaewitz, Martin Degeling, and Markus Dürmuth. 2021. Apps Against the Spread: Privacy Implications and User Acceptance of COVID-19-Related Smartphone Apps on Three Continents. In Proceedings of the 2021 CHI Conference on Human Factors in Computing Systems (Yokohama, Japan) (CHI '21). Association for Computing Machinery, New York, NY, USA, Article 70, 22 pages. https://doi.org/10.1145/3411764.3445517

[36] YouGov. 2020. YouGov COVID-19 tracker: government handling, France. (November 24, 2020). Retrieved August 16, 2021 from https://yougov.co.uk/ topics/international/articles-reports/2020/03/17/perception-government-handling-covid- 19.

[37] YouGov. 2020. YouGov COVID-19 tracker: government handling, Germany. (November 18, 2020). https://yougov.co.uk/topics/international/articlesreports/2020/03/17/perception-government-handling-covid-19. Retrieved August 16, 2021 from.

[38] Baobao Zhang, Sarah Kreps, Nina McMurry, and R. Miles McCain. 2020. Americans' perceptions of privacy and surveillance in the COVID-19 pandemic. Plos one 15, 12 (2020), e0242652.

Manuscript submitted to ACM 


\section{A APPENDIX - SEMI-STRUCTURED FOCUS GROUP GUIDE}

- Informed Consent

- Welcome

- WHO excerpt

- Q1: Have you ever used an official contact tracing app nationally approved by a health authority or the government?

- Q2: Why did you decide to [use | not use] a national contact tracing app?

- Q3: What do you see as the major benefits (if any) of using a contact tracing app?

- Q4: What do you see as the major drawbacks (if any) of using a contact tracing app?

- Q5: What features of the contact tracing app [do you | did you] use? What [do you | did you] value most about the app?

- Q6: When travelling abroad, would you be willing to install a contact tracing app approved by the public health authorities or government of the country where you are travelling to?

- Q7: Should a contact tracing app from your country be able to detect and share proximity contacts with other users of contact tracing apps, regardless of the app or its country of origin?

- Q8: Are there any privacy concerns that you have with respect to the use of national contact tracing apps?

- Debrief

\section{B APPENDIX - QUALITATIVE CODEBOOK}

We present our codebook with the first- and second-level codes as well as their respective counts below.

- adoption (48): active social context (1), avoiding misinformation (1), curiosity (2), personal safety (7), influence (1), request / mandate (1), security, privacy \& trust (10), society (21), technical capability (1), usefulness (3); • non-adoption (64): herd mentality (3), lack of efficiency (4), lack of interoperability (1), lack of promotion (1), lack of role models (2), lack of technical capability (1), distrust in the government (6), distrust in the developers (2), distrust in the technology (4), lack of usability (8), lack of utility (12), limited / lack of social interactions (4), privacy concerns (11), psychological discomfort (4), voluntary approach (1); • benefits (56): self-determination (16), useful features (1), efficiency (2), staying informed (4), identification of potential risks (11), mental and emotional comfort (4), help in modelling the spread (3), identification of infections (15); • drawbacks (47): potential repercussions (1), interoperability (1), costs and expenses (9), lack of usability (3), lack of functionality (1), lack of perceived effectiveness (13), privacy invasion (6), lack of clear information / communication (6), lack of perceived benefits (7); • features (7): requests (7); 